\title{
A new measure for the strength of electrical synapses
}

\author{
Julie S. Haas* \\ Department of Biological Sciences, Lehigh University, Bethlehem, PA, USA
}

Electrical synapses, like chemical synapses, mediate intraneuronal communication. Electrical synapses are typically quantified by subthreshold measurements of coupling, which fall short in describing their impact on spiking activity in coupled neighbors. Here, we describe a novel measurement for electrical synapse strength that directly evaluates the effect of synaptically transmitted activity on spike timing. This method, also applicable to neurotransmitter-based synapses, communicates the considerable strength of electrical synapses. For electrical synapses measured in rodent slices of the thalamic reticular nucleus and in simple model neurons, spike timing is modulated by tens of ms by activity in a coupled neighbor.

Keywords: gap junction, electrical synapses, efficacy, thalamic reticular nucleus, connexin36

\section{Introduction}

OPEN ACCESS

Edited by:

Tycho M. Hoogland,

Netherlands Institute

for Neuroscience, Netherlands

Reviewed by:

Marylka Uusisaari, Hebrew University of Jerusalem,

Israel

Ping Liu,

University of Connecticut Health

Center, USA

${ }^{*}$ Correspondence:

Julie S. Haas,

Department of Biological Sciences,

Lehigh University, 111 Research

Drive, Bethlehem, PA 18015, USA

julie.haas@/ehigh.edu

Received: 22 July 2015 Accepted: 11 September 2015 Published: 25 September 2015

Citation:

Haas JS (2015) A new measure for the strength of electrical synapses.

Front. Cell. Neurosci. 9:378.

doi: 10.3389/fncel.2015.00378
The strength of electrical synapses between gap junction-coupled neurons has traditionally been measured by the coupling coefficient (Bennett, 1966), which is the ratio of a steady, small voltage deflection transmitted from one cell to its neighbor across the synapse (Figure 1A). From the coupling coefficient, one can estimate the conductance of the synapse (Bennett, 1966; Fortier, 2010). Across the brain, average coupling coefficients measured from soma to soma vary from small $(<0.05)$ in inferior olive (Devor and Yarom, 2002) and hippocampus (Zsiros and Maccaferri, 2005); to moderate, 0.1-0.15, in the thalamic reticular nucleus (Landisman et al., 2002) and cortex (Gibson et al., 1999); to even larger values, 0.2 in MesV (Curti et al., 2012). Coupling coefficients for physiological signals such as spikelets (Galarreta and Hestrin, 2001; Haas and Landisman, 2012) have been measured (Figures 1B,C), but are typically smaller than those measured for steady voltage deflections, due to their faster timecourses.

Coupling coefficients do not describe the role of electrical synapses in spiking, which varies with many factors, including excitability and intercellular distance. Yet that role is often substantial: in the dorsal cochlear nucleus, fusiform to stellate cell coupling is so effective as to control spiking in the stellates (Apostolides and Trussell, 2013), and coupling was shown to sharply increase the probability of spiking in coupled hypothalamic cells of the cichlid fish (Ma et al., 2014). In other populations, the impact of electrical synapses may be diminished by distance from a dendrodendritic synapse to the somatic integrator, while still driving a dendritic spike (Trenholm et al., 2014). Supra-threshold measures for the strength of electrical synapses have been used: correlation coefficients can be computed and compared for coupled pairs (Galarreta and Hestrin, 1999; Gibson et al., 1999; Long et al., 2002; Blatow et al., 2003; Haas and Landisman, 2012; Ma et al., 2014). However, correlation-based measures require both neurons to be activated by other inputs to similar states of firing; that firing must be steady or periodic; and correlation is measured and averaged over a period of time, encompassing several to many spikes, conditions which are 
A
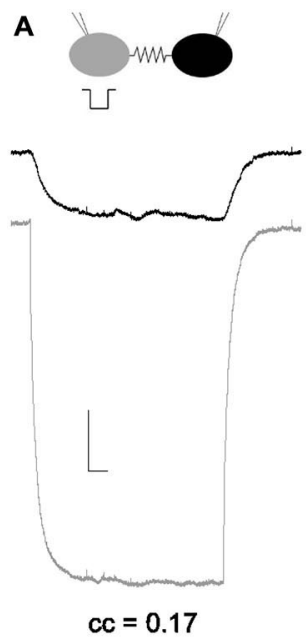

B

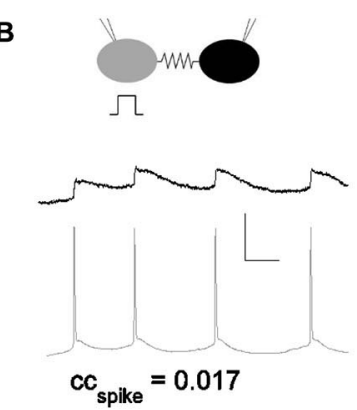

C

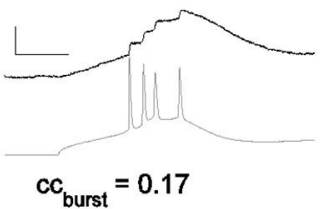

D

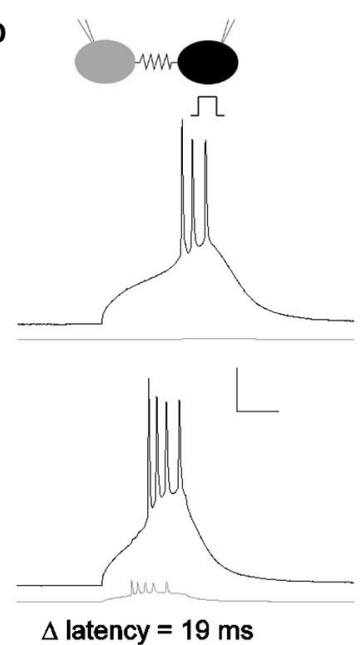

FIGURE 1 | Subthreshold and suprathreshold measurements for the strength of an electrical synapse. (A) Coupling measured with current pulses. Coupling coefficients are a ratio of voltage deflections. Here, voltage deflections were initiated by a step in current delivered in one neuron (gray) that echoed in the coupled neuron (black). $c c=0.17$ for the pair shown. Scale bar $1 \mathrm{mV}, 50 \mathrm{~ms}$. (B) Coupling coefficient measured by spike and spikelet amplitudes. Spikes were elicited in one neuron (gray) and spikelets in the coupled neighbor (black). Scale bar $2.5 \mathrm{mV}$ (black,) $25 \mathrm{mV}$ (gray), 25 ms. (C) Coupling coefficient measured by burst and burstlet amplitudes, for a longer burst event in one neuron (gray) and a burstlet in the coupled neighbor (black). Scale bar $2.5 \mathrm{mV}$ (black), $25 \mathrm{mV}$ (gray), $25 \mathrm{~ms}$. (D) Coupling measured by latency changes. Modulation of spike latency $\delta \mathrm{L}$ was measured by comparing timing of spikes elicited in one cell alone (black; gray cell quiet), and with the coupled neighbor also driven to spike (gray). Scale bar $2 \mathrm{mV}$ (gray), $20 \mathrm{mV}$ (black), $25 \mathrm{~ms}$. All data presented are from the same pair.

more the exception than the rule in vivo. The question remains: how strong is an electrical synapse, in the context of spiking neurons? Specifically, what is the impact of an electrical synapse on the fundamental unit of neuronal communicationa spike?

In order to both quantify the strength of an electrical synapse and to provide a better basis for comparison to chemical synapses, we introduce a novel measure, $\delta \mathrm{L}$, that expresses the efficacy of electrical synapses (Figure 1D). Herein, we explain the method and the simple test to measure it, compare it to traditionally used methods, and demonstrate its use in revealing the true strength of electrical synapses.

\section{Methods}

The data used here have been previously reported (Sevetson and Haas, 2014). Horizontal slices 350-400 $\mu \mathrm{m}$ thick were obtained from Sprague-Dawley rats aged P11 - P14 of either sex. Rats were anesthetized using isofluorane and euthanized in accordance with federal and Lehigh IACUC animal welfare guidelines. Slices were cut and incubated in sucrose solution (in mM): 72 Sucrose, $83 \mathrm{NaCl}, 2.5 \mathrm{KCl}, 1 \mathrm{NaPO}_{4}, 3.3 \mathrm{MgSO}_{4}$, $26.2 \mathrm{NaHCO}_{3}, 22$ dextrose, $0.5 \mathrm{CaCl}_{2}$. Slices were incubated at $36^{\circ} \mathrm{C}$ for $20 \mathrm{~min}$ and returned to room temperature until recording. The bath for solution for recording contained (in $\mathrm{mM}$ ): $126 \mathrm{NaCl}, 3 \mathrm{KCl}, 1.25 \mathrm{NaH}_{2} \mathrm{PO}_{4}, 2 \mathrm{MgSO}_{4}, 26 \mathrm{NaHCO}_{3}$, 10 dextrose and $2 \mathrm{CaCl}_{2}, 300-305 \mathrm{mOsm}$, saturated with 95\% $\mathrm{O}_{2} / 5 \% \mathrm{CO}_{2}$, The submersion recording chamber was held at $34^{\circ} \mathrm{C}$ (TC-324B, Warner Instruments). Micropipettes were filled with (in $\mathrm{mM}$ ): $135 \mathrm{~K}$-gluconate, $2 \mathrm{KCl}, 4 \mathrm{NaCl}$, 10 HEPES, 0.2 EGTA, 4 ATP-Mg, 0.3 GTP-Tris, and 10
phosphocreatine-Tris ( $\mathrm{pH}$ 7.25, 295 mOsm). For voltageclamp measurements, $135 \mathrm{mM} \mathrm{CsMSO}$ 年as substituted for K-gluconate. Either $1 \mathrm{M} \mathrm{CsOH}$ or $1 \mathrm{M} \mathrm{KOH}$ was used to adjust $\mathrm{pH}$ of the internal solution. The approximate bath flow rate was $2 \mathrm{ml} / \mathrm{min}$. Voltages are reported as corrected for the liquid junction potential and bridge-balanced. The TRN was visualized under $5 \times$, and pairs of TRN cells were identified at $40 \times$ IR-DIC optics (SliceScope, Scientifica). Signals were amplified and low-pass filtered at $8 \mathrm{kHz}$ (MultiClamp, Axon Instruments), digitized at $20 \mathrm{kHz}$ (lab-written Matlab routines controlling a National Instruments USB6221 DAQ board), and stored for offline analysis in Matlab (Mathworks, R2012a). Hodgkin-Huxley modeling was executed as previously described (Sevetson and Haas, 2014), simplified by setting calcium conductance to zero and using a single symmetrical electrical synapse. The three sodium conductances used were 60, 75 and $90 \mu \mathrm{S} / \mathrm{cm}^{2}$.

\section{Results}

In response to depolarizing input of increasing amplitude, neurons spike with decreasing latency. This is a fundamental, common property of neuronal response. Recording from dual whole-cell patches of coupled neurons in thalamic reticular nucleus in acute brain slices, we performed an experiment designed to compare spike times in a neuron minimally stimulated from rest, with and without input from a neighbor across an electrical synapse, a paradigm that is repeatable in almost any pair of excitable cells. 

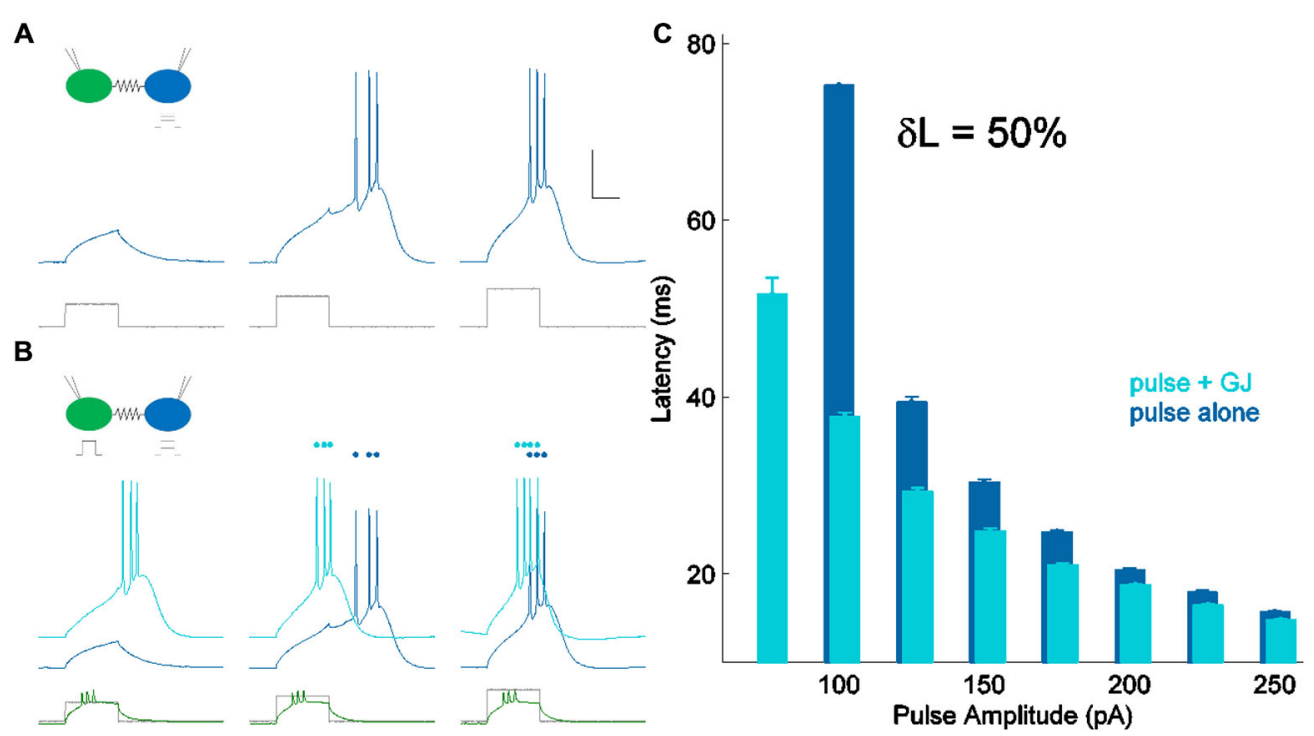

FIGURE 2 | Measuring $\delta$ L, latency modulation. (A) Spiking in one cell of a coupled pair (blue) in response to current pulses of increasing amplitude (lower, shown in gray). Scale bar $25 \mathrm{~ms}, 20 \mathrm{mV}$. The coupled cell was quiet and is not shown. (B) Spiking in the same cell (light blue) for the same current pulses as in (A) (lower, shown in gray), with the coupled neighbor also spiking (lower, shown in green). Responses from (A) are repeated, vertically offset for clarity (darker blue). (C) Latency of spiking in (A) (pulse alone) and (B) (pulse + GJ input) plotted against input amplitude. For peri-threshold inputs (100 pA) in this cell, $\delta \mathrm{L}$, the percentage change in perithreshold spike latency, was 50\%.

In all experiments, both cells were held near their resting voltage, at $-70 \mathrm{mV}$. We used a set of 10 current steps with maximum amplitude of approximately $1 \mathrm{pA}$ per $\mathrm{M} \Omega$ of input resistance, delivered to one cell of a coupled pair through the recording electrode. Thus, for a cell of input resistance $250 \mathrm{M} \Omega$, we delivered ten current pulses between 25 and $250 \mathrm{pA}$. We measured latency of spiking in that cell in two conditions: alone (Figure 2A), and with a suprathreshold input $(\sim 2 \times$ perithreshold) applied to the coupled neighbor (Figure 2B), driving it to spike before the first cell. Comparing the two sets of responses, we found that latency decreased when the synapse provided additional input (Figure 2C). We used this comparison to quantify the strength of the electrical synapse, for the smallest current step that consistently drove a spike in each cell. For the cell in Figure 2, the smallest input that reliably drove spiking was $100 \mathrm{pA}$; the cell did not spike for $75 \mathrm{pA}$ of input without GJ input and $\delta \mathrm{L}$ was not calculable. Over 36 neurons, peri-threshold inputs were $93.5 \pm$ $6.6 \mathrm{pA}$ (mean $\pm \mathrm{SEM}$ ). This value was chosen for latency comparison in order to provide a realistic measurement of perithreshold spike time modulation during synaptic input barrages in vivo.

Using these peri-threshold values of $\Delta t_{\text {alone }}$ as the latency of the spike with a quiet neighbor and $\Delta \mathrm{t}_{\text {paired }}$ as the latency of the
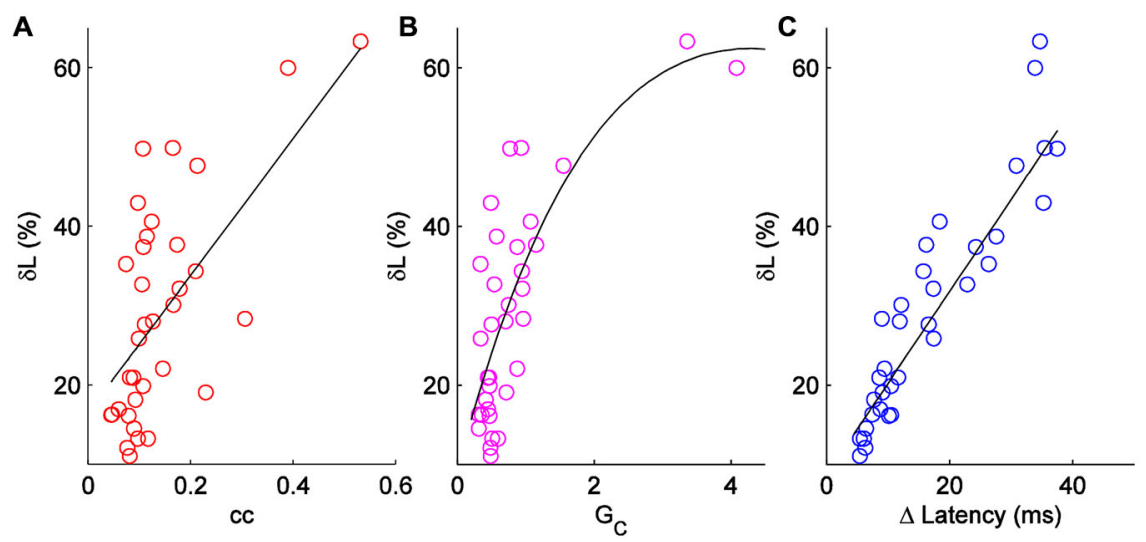

FIGURE 3 | Comparison of $\delta \mathbf{L}$ to other measures of electrical synapse strength. (A) $\delta \mathrm{L}$ plotted against coupling coefficient cc in each direction for a set of $n=18$ pairs. $R^{2}=0.38$. (B) $\delta \mathrm{L}$ plotted against coupling conductance $\mathrm{G}_{C} ; R^{2}=0.56$. (C) $\delta \mathrm{L}$ plotted against absolute change in latency for each cell in 18 pairs. 

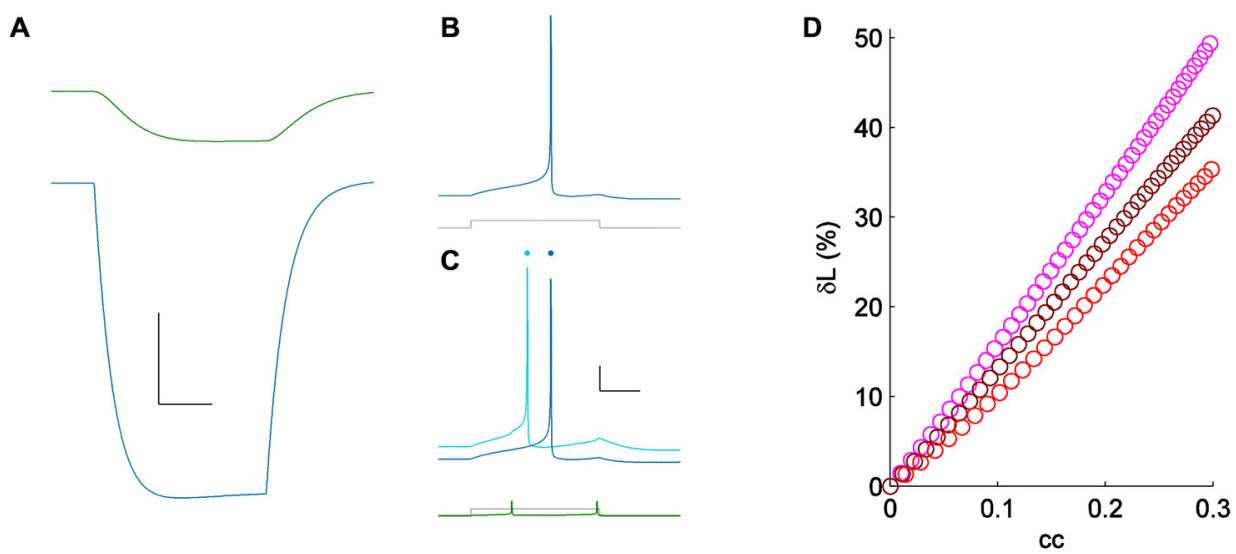

FIGURE 4 | (A) Coupling demonstrated by a hyperpolarizing current pulse in a pair of simple Hodgkin-Huxley neurons; $C C=0.15$. Scale bar $2 \mathrm{mV}$, $25 \mathrm{~ms}$. (B) Spiking one of the model cells (blue) for a minimal input (lower, gray); the coupled neuron was quiet. (C) Spiking in the same cell (light blue) for the same current pulses as in (A) (lower, shown in gray), with the coupled neighbor also spiking (lower, shown in green). Responses from (A) are repeated, vertically offset, for clarity (dark blue). Scale bar $10 \mathrm{mV}, 25 \mathrm{~ms}$. (D) $\delta \mathrm{L}$ plotted against coupling coefficient in the modeled pair, for three values of excitability [sodium conductances of 60 (pink), 75 (maroon) and 90 (red) $\left.\mu \mathrm{S} / \mathrm{cm}^{2}\right]$.

spike with an active neighbor, the quantity $\delta \mathrm{L}$ is expressed as a percentage change in latency:

$$
\delta \mathrm{L}=100 * \frac{\Delta t_{\text {alone }}-\Delta t_{\text {paired }}}{\Delta t_{\text {alone }}}
$$

$\delta \mathrm{L}$ has units of percentage in principle, $\delta \mathrm{L}$ can be negative. $\delta \mathrm{L}$ is unrelated to pulse input strength $\left(R^{2}=0.07\right.$; not shown). $\delta \mathrm{L}$ is moderately correlated to coupling coefficients, and better related to the coupling conductances (Figures 3A,B) measured by hyperpolarizing current inputs. While input from the electrical synapse often converted an input that, alone, was subthreshold into a supra-threshold input (e.g., $75 \mathrm{pA}$ in Figure 2C), $8 \mathrm{~L}$ does not include that effect. For our sample of electrical synapses, the average value of $\delta \mathrm{L}$ was $29.5 \pm 2.2 \%$ (mean \pm SEM, $n=36$; Figure 3C). Applied to the average peri-threshold latency in our dataset of $56 \mathrm{~ms}, \delta \mathrm{L}$ represents a difference in spike timing of $16.5 \mathrm{~ms}$, a substantial difference on a neuronal timescale.

$\delta \mathrm{L}$ is experimentally as simple to measure as coupling coefficients, and provides an output that directly describes the impact of electrical synapses. This method can also be applied to chemical synaptic inputs. In order to compare electrical to chemical synapses, we performed the same experiment on a pair of somatosensory cortical (layer II) cells that were coupled through a glutamatergic synapse, with average response amplitude of $0.45 \mathrm{mV}$, an average strength for a chemical synapses (Feldmeyer et al., 2005). For this excitatory chemical synapse, the average decrease in peri-threshold latency was $1.4 \mathrm{~ms}$, corresponding to a $\delta \mathrm{L}$ of $2.9 \%$. This comparison demonstrates that in the context of spiking, electrical synapses are an order of magnitude more powerful than excitatory chemical connections.

We quantified $\delta \mathrm{L}$ before and after depressing the synapse by coordinated induced bursting activity (Haas et al., 2011) in a coupled pair for which initially, $\delta \mathrm{L}$ was $19.6 \%$, representing a latency difference of $9.6 \pm 0.3 \mathrm{~ms}$ at baseline. Depression of the synapse by $10.2 \%$ increased $\delta \mathrm{L}$ by $12.2 \%$, to a final value of $22 \%$, representing change in perithreshold latency of 12.0 $\pm 0.6 \mathrm{~ms}(p<0.05)$. These values demonstrate that modest changes in electrical synaptic strength translate to physiologically meaningful changes in spike timing.

Because spiking in TRN neurons is heavily influenced by their low-threshold $\mathrm{T}$ current, we repeated measurement of $\delta \mathrm{L}$ in a coupled pair of simple Hodgkin-Huxley neurons (Figure 4). We used model neurons identical to those used in Sevetson and Haas (2014), but with zero $T$ conductance, reducing the model to only sodium and potassium currents with a linear and symmetrical electrical synapse. For minimal stimuli, we applied step inputs that yielded initial latencies of $\sim 75 \mathrm{~ms}$ (Figure 4B). For a moderate value of coupling ( $c c=0.15)$, activity across the electrical synapse accelerated the model neuron's spike time from $70-55 \mathrm{~ms}$, or $\delta \mathrm{L}$ of $21 \%$ (Figure $4 \mathrm{C}$ ). To test the dependence of neuronal excitability on $\delta \mathrm{L}$, we varied sodium conductance in the model by $25-50 \%$ (Figure $4 \mathrm{D}$ ). Using minimal stimuli in each set showed that while $\delta \mathrm{L}$ is weakly related to excitability, the strong modulatory effect of electrical synapses on spike times is reproduced by this simple model.

\section{Discussion}

The method of quantifying electrical synapse strength that we introduce here, $\delta \mathrm{L}$, reveals that electrical synapses are more powerful contributors to active spiking networks than subthreshold-based measurements of these synapses previously indicated. Together, this method and its application underline the strength of electrical synapses in shaping and altering spiking activity in coupled neurons across the brain.

By measuring the functional neuronal output, of spike times, $\delta \mathrm{L}$ captures interactions with or amplification of electrical synapses by postsynaptic nonlinear membrane conductances, 
such as the persistent sodium current (Curti et al., 2012; Haas and Landisman, 2012) or presynaptic effects, such as the afterhyperpolarizing currents relayed through gap junctions that delay spikes in coupled neighbors (Vervaeke et al., 2010). Like coupling coefficients, $\delta \mathrm{L}$ also can be used to quantify and compare asymmetry of electrical synapses (Sevetson and Haas, 2014).

In contrast to subthreshold-based measures, in principle $\delta \mathrm{L}$ can be measured by recording in a single neuron, while stimulating that neuron's coupled neighbors through other

\section{References}

Apostolides, P. F., and Trussell, L. O. (2013). Regulation of interneuron excitability by gap junction coupling with principal cells. Nat. Neurosci. 16, 1764-1772. doi: $10.1038 / \mathrm{nn} .3569$

Bennett, M. V. L. (1966). Physiology of Electrotonic Junctions. Ann. N. Y. Acad. Sci. 137, 509-539. doi: 10.1111/j.1749-6632.1966.tb50178.x

Blatow, M., Rozov, A., Katona, I., Hormuzdi, S. G., Meyer, A. H., Whittington, M. A., et al. (2003). A novel network of multipolar bursting interneurons generates theta frequency oscillations in neocortex. Neuron 38, 805-817. doi: 10.1016/s0896-6273(03)00300-3

Curti, S., Hoge, G., Nagy, J. I., and Pereda, A. E. (2012). Synergy between electrical coupling and membrane properties promotes strong synchronization of neurons of the mesencephalic trigeminal nucleus. J. Neurosci. 32, 4341-4359. doi: 10.1523/jneurosci.6216-11.2012

Devor, A., and Yarom, Y. (2002). Electrotonic coupling in the inferior olivary nucleus revealed by simultaneous double patch recordings. J. Neurophysiol. 87, 3048-3058.

Feldmeyer, D., Roth, A., and Sakmann, B. (2005). Monosynaptic connections between pairs of spiny stellate cells in layer 4 and pyramidal cells in layer $5 \mathrm{~A}$ indicate that lemniscal and paralemniscal afferent pathways converge in the infragranular somatosensory cortex. J. Neurosci. 25, 3423-3431. doi: 10. 1523/jneurosci.5227-04.2005

Fortier, P. A. (2010). Detecting and estimating rectification of gap junction conductance based on simulations of dual-cell recordings from a pair and a network of coupled cells. J. Theor. Biol. 265, 104-114. doi: 10.1016/j.jtbi.2010. 03.048

Galarreta, M., and Hestrin, S. (1999). A network of fast-spiking cells in the neocortex connected by electrical synapses. Nature 402, 72-75. doi: 10 . $1038 / 47029$

Galarreta, M., and Hestrin, S. (2001). Electrical synapses between GABAreleasing interneurons. Nat. Rev. Neurosci. 2, 425-433. doi: 10.1038/350 77566

Gibson, J. R., Beierlein, M., and Connors, B. W. (1999). Two networks of electrically coupled inhibitory neurons in neocortex. Nature 402, 75-79. doi: $10.1038 / 47035$

Haas, J. S., and Landisman, C. E. (2012). State-dependent modulation of gap junction signaling by the persistent sodium current. Front. Cell. Neurosci. 5:31. doi: $10.3389 /$ fncel.2011.00031 (extracellular or optogenetic) means. Thus, $\delta \mathrm{L}$ offers a way to detect and quantify electrical synapses that is less technically demanding than performing paired recordings.

\section{Funding}

JSH was supported in part as the Haddie Investigator, with a 2014 NARSAD Young Investigator Grant from the Brain and Behavior Research Foundation grant \#21343, and by the Whitehall Foundation grant \#2014-05-25.

Haas, J. S., Zavala, B., and Landisman, C. E. (2011). Activity-dependent long-term depression of electrical synapses. Science 334, 389-393. doi: 10.1126/science. 1207502

Landisman, C. E., Long, M. A., Beierlein, M., Deans, M. R., Paul, D. L., and Connors, B. W. (2002). Electrical synapses in the thalamic reticular nucleus. J. Neurosci. 22, 1002-1009.

Long, M. A., Deans, M. R., Paul, D. L., and Connors, B. W. (2002). Rhythmicity without synchrony in the electrically uncoupled inferior olive. J. Neurosci. 22, 10898-10905.

Ma, Y., Juntti, S. A., Hu, C. K., Huguenard, J. R., and Fernald, R. D. (2014). Electrical synapses connect a network of gonadotropin releasing hormone neurons in a cichlid fish. Proc. Natl. Acad. Sci. U. S. A. 112, 3805-3810. doi: 10. 1073/pnas.1421851112

Sevetson, J., and Haas, J. S. (2014). Asymmetry and modulation of spike timing in electrically coupled neurons. J. Neurophysiol. 113, 1743-1751. doi: 10.1152/jn. 00843.2014

Trenholm, S., McLaughlin, A. J., Schwab, D. J., Turner, M. H., Smith, R. G., Rieke, F., et al. (2014). Nonlinear dendritic integration of electrical and chemical synaptic inputs drives fine-scale correlations. Nat. Neurosci. 17, 1759-1766. doi: $10.1038 / \mathrm{nn} .3851$

Vervaeke, K., Lorincz, A., Gleeson, P., Farinella, M., Nusser, Z., and Silver, R. A. (2010). Rapid desynchronization of an electrically coupled interneuron network with sparse excitatory synaptic input. Neuron 67, 435-451. doi: 10. 1016/j.neuron.2010.06.028

Zsiros, V., and Maccaferri, G. (2005). Electrical coupling between interneurons with different excitable properties in the stratum lacunosum-moleculare of the juvenile CA1 rat hippocampus. J. Neurosci. 25, 8686-8695. doi: 10. 1523/jneurosci.2810-05.2005

Conflict of Interest Statement: The author declares that the research was conducted in the absence of any commercial or financial relationships that could be construed as a potential conflict of interest.

Copyright (C) 2015 Haas. This is an open-access article distributed under the terms of the Creative Commons Attribution License (CC BY). The use, distribution and reproduction in other forums is permitted, provided the original author(s) or licensor are credited and that the original publication in this journal is cited, in accordance with accepted academic practice. No use, distribution or reproduction is permitted which does not comply with these terms. 